\title{
LAGRANGIAN METHODS FOR OPTIMAL CONTROL PROBLEMS GOVERNED BY QUASI-HEMIVARIATIONAL INEQUALITIES
}

\author{
FENGZHEN LONG AND BIAO ZENG
}

Received 14 November, 2019

\begin{abstract}
The aim of this paper is to study an optimal control problem governed by a quasihemivariational inequality by using nonlinear Lagrangian methods. We first show the existence of solutions to the inequality problem, and then, we establish several sufficient conditions for the zero duality gap property between the optimal control problem and its nonlinear dual problem.
\end{abstract}

2010 Mathematics Subject Classification: 47J20; 49J20; 49N15

Keywords: quasi-hemivariational inequality, lagrangian method, optimal control, zero duality gap

\section{INTRODUCTION}

Optimal control problems governed variational inequalities have attracted great attention in recent years and have many applications in engineering and mechanics, see, for instance, $[1,5,20,25,27]$. Lagrangian methods are useful tools to study constrained optimization problems and optimal control problems. The zero duality gap property in the study of optimization problems, where the optimal values of the prime and dual problems equal, is an important property to be used in the development of primal-dual methods. For more details on the zero duality gap property for nonconvex optimization problems, we refer to [21,32] and the references therein. Recently, Zhou et al. [31] investigated the zero duality gap property for an optimal control problem governed by a variational inequality and gave several sufficient conditions for the zero duality gap property between the optimal control problem and its nonlinear Lagrangian dual problem. Later, Wang et al. [24] generalized the results in [31] and studied an optimal control problem where the state of the system is defined by a mixed quasi-variational inequality.

In this work, we study a class of quasi-hemivariational inequalities. The hemivariational inequalities were introduced by Panagiotoulos $[17,18]$ for describing a variety of mechanical problems such as unilateral contact in nonlinear elasticity, adhesive and friction effects, nonconvex semipermeability, masonry structures, and delamination in multilayered composites. Quasi-hemivariational inequalities are important 
and useful generalization of variational inequalities and hemivariational inequalities, and have significant applications in mechanics problems (see $[12,13])$. Very recently, many authors studied the existence results for some types of hemivariational inequalities (see $[8,15,23,26,29,30])$. For some related optimal control problem for hemivariational inequalities, we refer to [4, 6, 7, 9-11,14, 19,22].

The purpose of this paper is to study the optimal control by a quasi-hemivariational inequality. We first show the set of the solutions to the variational inequality is nonempty. Then we obtain several sufficient conditions for the zero duality gap property between the optimal control problem and its nonlinear dual problem. The main novelties of the paper are following. Instead of equilibrium problems and variational inequality problems widely used in the literature, we deal with a quasihemivariational inequality problem. The results obtained in this paper improve and extend many results in equilibrium problems and variational inequalities. To our best knowledge, it is the first work to study the zero duality gap property between the optimal control problem and its nonlinear dual problem for hemivariational inequalities. Several hemivariational inequality problems can also be further studied.

The rest of this paper is organized as follows. In the next section, we will introduce some useful preliminaries and necessary materials. In Section 3, we show the existence of solutions to the quasi-hemivariational inequality. In Section 4, we provide a result for the zero duality gap property between the optimal control problem and its nonlinear dual problem. Theorem 2 and Theorem 3 are main results in this part. We also give some deduced theorems in which we consider a special case of quasi-hemivariational inequalities in the last section.

\section{PRELIMinaries}

Let $\left(X,\|\cdot\|_{X}\right)$ be a Banach space, $X^{*}$ denote its dual space and $\langle\cdot, \cdot\rangle_{X}$ be the duality pairing between $X^{*}$ and $X$. We denote by " $\rightarrow$ " the strong convergence and by " $\rightarrow$ " the weak convergence.

Definition 1. A function $f: X \rightarrow \mathbb{R}$ is said to be

(i) (weakly) upper semicontinuous (u.s.c.) at $u_{0}$, if any sequence $\left\{u_{n}\right\} \subset X$ with $\left(u_{n} \rightarrow u_{0}\right) u_{n} \rightarrow u_{0}$, we have $\limsup f\left(u_{n}\right) \leq f\left(u_{0}\right)$.

(ii) (weakly) lower semicontinuous (1.s.c.) at $u_{0}$, if any sequence $\left\{u_{n}\right\} \subset X$ with $\left(u_{n} \rightarrow u_{0}\right) u_{n} \rightarrow u_{0}$, we have $f\left(u_{0}\right) \leq \liminf f\left(u_{n}\right)$.

(iii) $f$ is said to be (weakly) u.s.c. (1.s.c.) on $X$, if $f$ is (weakly) u.s.c. (l.s.c.) at $u$ for all $u \in X$.

Definition 2 ([28]). Let $D$ be a nonempty subset of $X$ and let $F: D \rightarrow X^{*}$ be a single-valued mapping. $F$ is said to be

(1) continuous if for every $u_{0} \in D$ and any sequence $\left\{u_{n}\right\} \subset D$ with $u_{n} \rightarrow u_{0}$, we have $F u_{n} \rightarrow F u_{0}$. 
(2) hemicontinuous if for all $u, v, w \in D$ the functional, $t \rightarrow\langle F(u+t v), z\rangle_{X}$ is continuous on $[0,1]$.

(3) strongly continuous if for every $u_{0} \in D$ and any $\left\{u_{n}\right\} \subset D$ with $u_{n} \rightarrow u_{0}$ in $D$, we have $F u_{n} \rightarrow F u_{0}$.

(4) monotone if for any $u_{1}, u_{2} \in D$, we have

$$
\left\langle F u_{1}-F u_{2}, u_{1}-u_{2}\right\rangle_{X} \geq 0 .
$$

(5) pseudomonotone if for every $u_{0} \in D$ and any $\left\{u_{n}\right\} \subset D$ with $u_{n} \rightarrow u_{0}$ and $\limsup \left\langle F u_{n}, u_{n}-u_{0}\right\rangle_{X} \leq 0$, we have

$$
\left\langle F u_{0}, u_{0}-v\right\rangle_{X} \leq \liminf _{n \rightarrow \infty}\left\langle F u_{n}, u_{n}-v\right\rangle_{X}
$$

for all $v \in D$.

Remark 1 ([28]). (1) If $F$ is hemicontinuous and monotone, then $F$ is pseudomonotone.

(2) If $F: D \subset X \rightarrow X^{*}$ and is hemicontinuous if and only if $F$ is continuous from the topology of $X$ to the weakly* topology of $X^{*}$.

(3) Let $F_{1}, F_{2}: D \rightarrow X^{*}$ be operators. If $F_{1}$ is pseudomonotone and $F_{2}$ is strongly continuous, then $F_{1}+F_{2}$ is pseudomonotone. In addition, if $F_{1}$ and $F_{2}$ are pseudomonotone, then $F_{1}+F_{2}$ is also pseudomonotone.

Definition 3. Let $G: X_{1} \rightarrow 2^{X_{2}}$ be a multivalued mapping from a topological space $X_{1}$ into a topological space $X_{2}$. $G$ is said to be bounded if $G(D)$ is a bounded set for any bounded subset $D$ of $X_{1}$.

Definition 4 ([16]). Let $D$ be a nonempty subset of $X$ and $K: D \rightarrow 2^{X}$ be a multivalued mapping. For any $\left\{w_{n}\right\} \subset D$ with $w_{n} \rightarrow w_{0} \in D$, we say that the sequence of sets $K\left(w_{n}\right)$ Mosco-converges to $K(w)$ if the following two conditions hold:

(i) for every sequence $\left\{u_{n}\right\}$, where $u_{n} \in K\left(w_{n}\right)$, such that $u_{n} \rightarrow u_{0}$, then $u_{0} \in$ $K\left(w_{0}\right)$;

(ii) for every $u_{0} \in K\left(w_{0}\right)$, there exists $u_{n} \in K\left(w_{n}\right)$ (for $n$ large enough) such that $u_{n} \rightarrow u_{0}$.

Lemma 1 ([3]). Let $D$ be a nonempty subset of a reflexive Banach space $X$, and let $G: D \rightarrow 2^{X}$ be a multivalued mapping satisfying

(a) $G$ is a KKM mapping, that is, for any $\left\{v_{1}, v_{2}, \ldots, v_{n}\right\} \subset D$, the convex hull $\operatorname{co}\left\{v_{1}, v_{2}, \cdots, v_{n}\right\}$ is contained in $\bigcup_{i=1}^{n} G\left(v_{i}\right)$,

(b) $G(v)$ is closed in $X$ for every $v \in D$,

(c) $G\left(v_{0}\right)$ is compact in $X$ for some $v_{0} \in D$.

Then $\bigcap_{v \in D} G(v) \neq \varnothing$.

Next, we present some needed elements of subdifferential calculus for locally Lipschitz functions (see [2,12]). 
Given a locally Lipschitz function $J: X \rightarrow \mathbb{R}$ on a Banach space $X$, we denote by $J^{\circ}(u, v)$ the generalized directional derivative of $J$ at the point $u \in X$ in the direction $v \in X$, that is

$$
J^{\circ}(u ; v):=\limsup _{\lambda \rightarrow 0^{+} ; w \rightarrow u} \frac{J(w+\lambda v)+J(w)}{\lambda} .
$$

The generalized gradient of $J: X \rightarrow \mathbb{R}$ at $u \in X$ is defined by

$$
\partial J(u):=\left\{\xi \in X^{*}: J^{\circ}(u ; v) \geq\langle\xi ; v\rangle_{X} \text { for all } v \in X\right\} .
$$

Proposition 1 ([2]). Let $J: X \rightarrow \mathbb{R}$ be locally Lipschitz of rank $L_{u}>0$ near $u \in X$. Then, there hold the following:

(a) the function $v \rightarrow J^{\circ}(u ; v)$ is positively homogeneous, subadditive and satisfies $\left|J^{\circ}(u ; v)\right| \leq L_{u}\|v\|_{X}$ for all $v \in X$;

(b) $J^{\circ}(u ; v)$ is u.s.c. as a function of $(u, v)$;

(c) $\partial J(u)$ is a nonempty, convex, weak ${ }^{*}$ compact subset of $X^{*}$ with $\|\xi\|_{X^{*}} \leq L_{u}$ for all $\xi \in \partial J(u)$;

(d) for each $v \in X$, we have $J^{\circ}(u ; v)=\max \left\{\langle\xi, v\rangle_{X}: \xi \in \partial J(u)\right\}$.

Next, we introduce the framework of this paper. We assume that $X, V$ and $E$ are reflexive Banach spaces, $U$ is a nonempty closed convex subset of $V$ and $K$ : $U \rightarrow 2^{X}$ is a multivalued mapping with nonempty values. Let $\mathcal{I}: X \times V \rightarrow \mathbb{R}$ and $A: X \rightarrow X^{*}, B: U \rightarrow X^{*}, J: E \rightarrow \mathbb{R}, \gamma: X \rightarrow E$ be given mappings, $f: X \times X \rightarrow \overline{\mathbb{R}}:=$ $\mathbb{R} \cup\{ \pm \infty\}$ be a function such that $f(u, u)=0$ for every $u \in X$. Consider the following optimal control problem governed by a quasi-variational inequality: Find $w^{*} \in U$ and $u \in S\left(w^{*}\right)$ such that

$$
\left\{\begin{array}{l}
\min \mathcal{J}(u, w) \\
\text { subject to }(w, u) \in U \times S(w),
\end{array}\right.
$$

where $S(w)$ is the set of solutions to the following quasi-hemivariational inequality corresponding to $w$ : find $u \in K(w)$ such that

$$
\langle A u+B w, v-u\rangle_{X}+f(u, v)+J^{\circ}(\gamma u ; \gamma v-\gamma u) \geq 0, \quad \forall v \in K(w) .
$$

For any $w \in U$, we define

$$
\begin{gathered}
g_{w}(u)=\sup _{v \in K(w)}\langle A u+B w, v-u\rangle_{X}+f(u, v)+J^{\circ}(\gamma u ; \gamma v-\gamma u), \\
K_{w}(y)=\left\{u \in K(w): g_{w}(u) \leq y\right\} .
\end{gathered}
$$

Thus, $g_{w}(u) \geq 0$ and $K_{w}(0)=\left\{u \in K(w): g_{w}(u)=0\right\}=S(w)$. Define the perturbation function $\beta$ as

$$
\beta(y)=\inf _{w \in U, u \in K_{w}(y)} \mathcal{J}(w, u) .
$$

It is easy to see that $\beta(0)$ is the optimal value of problem (2.1). 
Definition 5 ([1]). Let $\mathbb{R}_{+}=[0,+\infty)$ and $P: \mathbb{R} \times \mathbb{R}_{+} \rightarrow \mathbb{R}$ be a function. A nonlinear Lagrangian function $L_{p}: U \times K(U) \times \mathbb{R}_{+} \rightarrow \mathbb{R}$ for problem (2.1) is defined as

$$
L_{P}(w, u, d)=P\left(\mathcal{J}(w, u), d g_{w}(u)\right) .
$$

The function

$$
F_{P}(d)=\inf _{w \in U, u \in K(w)} L_{P}(w, u, d)
$$

is called its nonlinear Lagrangian dual function. The equality

$$
\beta(0)=\sup _{d \in \mathbb{R}_{+}} F_{P}(d)
$$

is called the zero duality gap property.

Assumption 1. ( $\left.P_{i}\right)$ If $y_{1} \leq y_{2}$, then $P\left(y_{1}, z\right) \leq P\left(y_{2}, z\right), \forall z \in \mathbb{R}_{+}$.

$\left(P_{i i}\right) p(y, 0)=y, \forall y \in \mathbb{R}$.

$\left(P_{i i i}\right)$ There exists a number $a>0$, such that $P(y, z) \geq \max \{y, a z\}, \forall(y, z) \in \mathbb{R} \times \mathbb{R}_{+}$.

Lemma 2 ([21]). Suppose that $P$ is a continuous function satisfying $P_{i}$ and $P_{i i}$. If the zero duality gap property (2.6) holds, then the perturbation function $\beta$ is l.s.c. at the origin.

Lemma 3 ([21]). Suppose that $P$ satisfies $\left(P_{i}\right),\left(P_{i i}\right),\left(P_{i i i}\right)$. If $-\infty<\beta(0)<+\infty$ and the perturbation function $\beta$ is l.s.c. at the origin, then the zero duality gap property (2.6) holds.

\section{EXISTENCE OF SOLUTIONS}

In this section, we give an existence result for the quasi-hemivariational inequality (2.2).

Consider the following hypotheses on the data of (2.2).

$\left(H_{K}\right): K: U \rightarrow 2^{X}$ is such that for each $w \in U, 0 \in K(w)$ and the set $K(w)$ is closed and convex in $X$.

$\left(H_{A}\right): A: K(U) \rightarrow X^{*}$ is hemicontinuous and monotone.

$\left(H_{f}\right): f: X \times X \rightarrow \overline{\mathbb{R}}$ is a mapping satisfying the following conditions:

(i) $\mathcal{D}(f)=\{u \in K(U): f(u, v) \neq-\infty, \forall v \in K(U)\}$ is nonempty,

(ii) $f(u, u)=0$ for all $u \in K(U)$,

(iii) $f(u, v)+f(v, u)=0$ for all $u, v \in K(U)$,

(iv) for every $v \in K(U), f(\cdot, v)$ is weakly u.s.c.,

(v) for every $u \in K(U), f(u, \cdot)$ is convex.

$\left(H_{J}\right): J: E \rightarrow \mathbb{R}$ is a locally Lipschitz function.

$\left(H_{\gamma}\right): \gamma: X \rightarrow E$ is a linear, bounded and compact operator.

$\left(H_{0}\right):$

$$
\lim _{u \in X,\|u\|_{X} \rightarrow \infty} \frac{\langle A u, u\rangle_{X}+\inf _{\eta_{u} \in \partial J(\gamma u)}\left\langle\eta_{u}, \gamma u\right\rangle_{E}+f(0, u)}{\|u\|_{X}}=+\infty .
$$


Theorem 1. Assume that $\left(H_{K}\right),\left(H_{A}\right),\left(H_{f}\right),\left(H_{J}\right),\left(H_{\gamma}\right),\left(H_{0}\right)$ are satisfied. Then $S(w) \neq \varnothing$.

Proof. (i) At first, we suppose that $K(w)$ is bounded in $X$. Introduce the multivalued mapping $G: K(w) \rightarrow 2^{K(w)}$ as follows:

$G(v):=\left\{u \in K(w):\langle A v+B w, v-u\rangle_{X}+f(u, v)+J^{\circ}(\gamma u ; \gamma v-\gamma u) \geq 0\right\}, \forall v \in K(w)$.

We claim that $G(v)$ is weakly closed in $V$. If for $\left\{u_{n}\right\} \subset G(v)$ one has $u_{n} \rightarrow u$, then $u \in K(w)$ and, for each $n \in \mathbb{N}$

$$
\left\langle A v+B w, v-u_{n}\right\rangle_{X}+f\left(u_{n}, v\right)+J^{\circ}\left(\gamma u_{n} ; \gamma v-\gamma u_{n}\right) \geq 0 .
$$

Proposition $1(b)$ and $\left(H_{f}\right)$ (iii) imply

$$
\begin{aligned}
0 & \leq \limsup _{n \rightarrow \infty}\left\langle A v+B w, v-u_{n}\right\rangle_{X}+f\left(u_{n}, v\right)+J^{\circ}\left(\gamma u_{n} ; \gamma v-\gamma u_{n}\right) \\
& \leq\langle A v+B w, v-u\rangle_{X}+f(u, v)+J^{\circ}(\gamma u ; \gamma v-\gamma u),
\end{aligned}
$$

which proves that $u \in G(v)$, thus $G(v)$ is weakly closed in $V$. We continue with the proof by arguing separately in two cases: (a) $G$ is a KKM mapping. (b) $G$ is not a KKM mapping. Assume case (a). Since $K(w)$ is bounded, closed and convex in the reflexive Banach space $V$, it is weakly compact. Due to the assertion above, for every $v \in K(w) G(v)$ is weakly compact too. Lemma 1 with respect to the weak topology of $X$ yields

$$
\bigcap_{v \in K(w)} G(v) \neq \varnothing .
$$

Taking $u_{0} \in \bigcap_{v \in K(w)} G(v)$, we have

$$
\left\langle A v+B w, v-u_{0}\right\rangle_{X}+f\left(u_{0}, v\right)+J^{\circ}\left(\gamma u_{0} ; \gamma v-\gamma u_{0}\right) \geq 0, \quad \forall v \in K(w) .
$$

Let an arbitrary $v^{\prime} \in K(w)$ and let $v_{n}:=\frac{1}{n} v^{\prime}+\left(1-\frac{1}{n}\right) u_{0} \in K(w)$. Then we have

$$
\left\langle A v_{n}+B w, v_{n}-u_{0}\right\rangle_{X}+f\left(u_{0}, v_{n}\right)+J^{\circ}\left(\gamma v_{n} ; \gamma v_{n}-\gamma u_{0}\right) \geq 0 .
$$

Then

$$
\begin{aligned}
0 \leq & \left\langle A v_{n}+B w, \frac{1}{n} v^{\prime}+\left(1-\frac{1}{n}\right) u_{0}-u_{0}\right\rangle_{X}+f\left(u_{0}, \frac{1}{n} v^{\prime}+\left(1-\frac{1}{n}\right) u_{0}\right) \\
& +J^{\circ}\left(\gamma v_{n} ; \gamma-v^{\prime}+\left(1-\frac{1}{n}\right) u_{0}-\gamma u_{0}\right) \\
\leq & \frac{1}{n}\left[\left\langle A v_{n}+B w, v^{\prime}-u_{0}\right\rangle_{X}+f\left(u_{0}, v^{\prime}\right)+J^{\circ}\left(\gamma v_{n} ; \gamma v^{\prime}-\gamma u_{0}\right)\right] .
\end{aligned}
$$

Multiplying the last inequality by $n$ and letting $n \rightarrow \infty$, we have

$$
\left\langle A u_{0}+B w, v^{\prime}-u_{0}\right\rangle_{X}+f\left(u_{0}, v^{\prime}\right)+J^{\circ}\left(\gamma u_{0} ; \gamma v^{\prime}-\gamma u_{0}\right)>0, \quad \forall v^{\prime} \in K(w),
$$

which concludes $u_{0} \in S(w)$. 
Now, admit case (b). Then, one can find $v_{1}, v_{2}, \ldots, v_{N} \in K(w)$ and $u_{0}=\sum_{j=1}^{N} \lambda_{j} v_{i}$ with $\lambda_{i} \in[0,1]$ and $\sum_{j=1}^{N} \lambda_{i}=1$ such that

$$
u_{0} \nsubseteq \bigcup_{j=1}^{N} G\left(v_{j}\right),
$$

which expresses that

$$
\left\langle A v_{j}+B w, v_{j}-u_{0}\right\rangle_{X}+f\left(u_{0}, v_{j}\right)+J^{\circ}\left(\gamma u_{0} ; \gamma v_{j}-\gamma u_{0}\right)<0 .
$$

Claim. There exists a neighborhood $O$ of $u_{0}$ in $X$ such that whenever $v \in O \cap K(w)$, there holds

$$
\left\langle A v_{j}+B w, v_{j}-v\right\rangle_{X}+f\left(v, v_{j}\right)+J^{\circ}\left(\gamma v ; \gamma v_{j}-\gamma v\right)<0 .
$$

Arguing by contradiction, let us assume that there exist $u_{n} \in K(w)$ and $j_{n} \in\{1,2, \ldots, N\}$ such that $u_{n} \rightarrow u_{0}$ and, for each $n$,

$$
\left\langle A v_{j_{n}}+B w, v_{j_{n}}-u_{n}\right\rangle_{X}+f\left(u_{n}, v_{j_{n}}\right)+J^{\circ}\left(\gamma u_{n} ; \gamma v_{j_{n}}-\gamma u_{n}\right) \geq 0 .
$$

Then, there exists $j_{0} \in\{1,2, \ldots, N\}$ such that for all $n$

$$
\left\langle A v_{j_{n}}+B w, v_{j_{0}}-u_{n}\right\rangle_{X}+f\left(u_{n}, v_{j_{0}}\right)+J^{\circ}\left(\gamma u_{n} ; \gamma v_{j_{0}}-\gamma u_{n}\right) \geq 0 .
$$

Passing to the limit gives

$$
\begin{aligned}
0 & \leq \limsup _{n \rightarrow \infty}\left\langle A v_{j_{0}}+B w, v_{j_{0}}-u_{n}\right\rangle_{X}+f\left(u_{n}, v_{j_{0}}\right)+J^{\circ}\left(\gamma u_{n} ; \gamma v_{j_{0}}-\gamma u_{n}\right) \\
& \leq\left\langle A v_{j_{0}}+B w, v_{j_{0}}-u_{0}\right\rangle_{X}+f\left(u_{0}, v_{j_{0}}\right)+J^{\circ}\left(\gamma u_{n} ; \gamma v_{j_{0}}-\gamma u_{0}\right),
\end{aligned}
$$

which contradicts (3.2). The claim is proven.

Using Claim, we have for each $j \in\{1,2, \ldots, N\}$ that

$$
\left\langle A v_{j}+B w, v_{j}-v\right\rangle_{X}+f\left(v, v_{j}\right)+J^{\circ}\left(\gamma v ; \gamma v_{j}-\gamma v\right)<0, \quad \forall v \in O \bigcap K(w) .
$$

It follows from the monotonicity of $A$ that

$$
\left\langle A v+B w, v_{j}-v\right\rangle_{X}+f\left(v, v_{j}\right)+J^{\circ}\left(\gamma v ; \gamma v_{j}-\gamma v\right) \leq 0 .
$$

Furthermore, on the basis of Proposition 2.13(d) and the convexity of $f(v, \cdot)$, we note that

$$
\begin{aligned}
& \left\langle A v+B w, v-u_{0}\right\rangle_{X}+f\left(v, u_{0}\right)+J^{\circ}\left(\gamma v ; \gamma u_{0}-\gamma v\right) \\
= & \left\langle A v+B w, \sum_{j=1}^{N} \lambda_{j} v_{j}-v\right\rangle_{X}+f\left(v, \sum_{j=1}^{N} \lambda_{j} v_{j}\right)+J^{\circ}\left(\gamma v ; \gamma u_{0}-\gamma v\right) \\
\leq & \sum_{j=1}^{N} \lambda_{j}\left[\left\langle A v+B w, v_{j}-v\right\rangle_{X}+f\left(v, v_{j}\right)+J^{\circ}\left(\gamma v ; \gamma v_{j}-\gamma v\right)\right] \\
\leq & 0
\end{aligned}
$$


for all $v \in O \bigcap K(w)$. Since

$$
J^{\circ}\left(\gamma v ; \gamma u_{0}-\gamma v\right)+J^{\circ}\left(\gamma v ; \gamma v-\gamma u_{0}\right) \geq J^{\circ}(\gamma v ; \gamma 0)=0,
$$

we obtain

$$
\left\langle A v+B w, v-u_{0}\right\rangle_{X}+f\left(u_{0}, v\right)+J^{\circ}\left(\gamma v ; \gamma v-\gamma u_{0}\right)>0, \quad \forall v \in O \bigcap K(w) .
$$

To prove that $u_{0} \in S(w)$, let an arbitrary $y \in K(w)$ and consider $y_{n}:=\frac{1}{n} y+\left(1-\frac{1}{n}\right) u_{0} \in$ $O \bigcap K(w)$. Hence, (3.3) can be used to obtain

$$
\left\langle A y_{n}+B w, y_{n}-u_{0}\right\rangle_{X}+f\left(u_{0}, y_{n}\right)+J^{\circ}\left(\gamma y_{n} ; \gamma y_{n}-\gamma u_{0}\right) \geq 0 .
$$

Then

$$
\begin{aligned}
0 \leq & \left\langle A y_{n}+B w, \frac{1}{n} y+\left(1-\frac{1}{n}\right) u_{0}-u_{0}\right\rangle_{X}+f\left(u_{0}, \frac{1}{n} y+\left(1-\frac{1}{n}\right) u_{0}\right) \\
& +J^{\circ}\left(\gamma y_{n} ; \gamma\left(\frac{1}{n} y+\left(1-\frac{1}{n}\right) u_{0}\right)-\gamma u_{0}\right) \\
\leq & \frac{1}{n}\left[\left\langle A y_{n}+B w, y-u_{0}\right\rangle_{X}+f\left(u_{0}, y\right)+J^{\circ}\left(\gamma y_{n} ; \gamma y-\gamma u_{0}\right)\right] .
\end{aligned}
$$

Multiplying the last inequality by $n$ and letting $n \rightarrow \infty$, we have

$$
\left\langle A u_{0}+B w, y-u_{0}\right\rangle_{X}+f\left(u_{0}, y\right)+J^{\circ}\left(\gamma u_{0} ; \gamma y-\gamma u_{0}\right)>0, \quad \forall y \in K(w),
$$

which concludes $u_{0} \in S(w)$.

(ii) Now, we assume that $K(w)$ is unbounded. For any given $w \in U$, letting $B_{r}=$ $\{v \in X:\|v\| \leq r\}$ and $K^{r}=B_{r} \bigcap K(w)$, we get that $K^{r}$ is a bounded, closed and convex subset of $X$. According to the assumptions, Remark 1 implies that $A+B$ is demicontinuous and pseudomonotone. By (i), we know there exists $u_{r} \in K^{r}$ such that

$$
\left\langle A u_{r}+B w, v-u_{r}\right\rangle+f\left(u_{r}, v\right)+J^{\circ}\left(\gamma u_{r} ; \gamma v-\gamma u_{r}\right) \geq 0, \quad \forall v \in K^{r} .
$$

In particular, taking $v=0$ in (3.4), there exists $u_{r} \in K^{r}$ such that

$$
\left\langle A u_{r}+B w, u_{r}\right\rangle-f\left(u_{r}, 0\right)-J^{\circ}\left(\gamma u_{r} ; \gamma 0-\gamma u_{r}\right) \leq 0, \quad \forall v \in K^{r} .
$$

it follows from $\left(H_{0}\right)$ that $\left\{u_{r}\right\}$ is bounded. So $\left\|u_{r}\right\| \leq M$ for some real number $M>0$. Let $r=M+1$. For each $v \in K(w)$, we can choose $t \in(0,1)$ small enough such that $v_{r}=u_{r}+t\left(v-u_{r}\right) \in K^{r}$. Substituting $v_{r}$ into (3.4), we obtain that

$$
\begin{gathered}
\left\langle A u_{r}+B w, u_{r}+t\left(v-u_{r}\right)-u_{r}\right\rangle+f\left(u_{r}, u_{r}+t\left(v-u_{r}\right)\right) \\
\left.+J^{\circ}\left(\gamma u_{r} ; \gamma\left(u_{r}+t\left(v-u_{r}\right)\right)-\gamma u_{r}\right)\right\rangle \geq 0 .
\end{gathered}
$$

Then

$$
\begin{aligned}
0 \leq & \left\langle A u_{r}+B w, u_{r}+t\left(v-u_{r}\right)-u_{r}\right\rangle+f\left(u_{r}, u_{r}+t\left(v-u_{r}\right)\right) \\
& \left.+J^{\circ}\left(\gamma u_{r} ; \gamma\left(u_{r}+t\left(v-u_{r}\right)\right)-\gamma u_{r}\right)\right\rangle \\
\leq & t\left[\left\langle A u_{r}+B w, v-u_{r}\right\rangle+f\left(u_{r}, v\right)+J^{\circ}\left(\gamma u_{r} ; \gamma v-\gamma u_{r}\right)\right] .
\end{aligned}
$$


Dividing by $t$, it follows that

$$
\left\langle A u_{r}+B w, v-u_{r}\right\rangle+f\left(u_{r}, v\right)+J^{\circ}\left(\gamma u_{r} ; \gamma v-\gamma u_{r}\right) \geq 0, \quad \forall v \in K(w),
$$

and hence $u_{r} \in S(w)$. The proof is completed.

Remark 2. From the theorem above, it is clear that hypothesis $\left(H_{0}\right)$ can be omitted if $K$ is bounded.

\section{OPTIMAL CONTROL}

In this section, we establish several conditions to guarantee the zero duality gap property for the optimal control problem governed by quasi-hemivariational inequalities and its dual problem.

Theorem 2. Assume that $\left(H_{K}\right),\left(H_{A}\right),\left(H_{f}\right),\left(H_{J}\right),\left(H_{\gamma}\right)$ are satisfied. Suppose that, in addition, $K: U \rightarrow 2^{X}$ is bounded, $A: K(U) \rightarrow X^{*}$ is bounded, $f(u, \cdot)$ is u.s.c. for every $u \in K(U), \mathcal{J}: U \times K(U) \rightarrow \mathbb{R}$ is weakly l.s.c. function, $B: U \rightarrow X^{*}$ is strongly continuous from the weak topology of $V$ to the topology of $X^{*}$ and the following conditions are satisfied

(i) for any $w \in U, u \in K(w), \lim _{\|w\| \rightarrow+\infty} \mathcal{I}(w, u)=+\infty$,

(ii) for all $w_{n} \in U$ with $w_{n} \rightarrow w, K\left(w_{n}\right)$ Mosco-converges to $K(w)$.

Then $-\infty<\beta(0)<+\infty$.

Proof. From Theorem 1, it follows that for each $w \in U, S(w) \neq \varnothing$. Let $\left\{\left(w_{n}, u_{n}\right)\right\} \subset$ $U \times K(U)$ be a sequence satisfying $u_{n} \in S\left(w_{n}\right)$ such that

$$
\mathcal{I}\left(w_{n}, u_{n}\right) \leq \inf _{w \in U, u \in S(w)} \mathcal{I}(w, u)+\frac{1}{n}=\beta(0)+\frac{1}{n}, \quad n=1,2, \ldots .
$$

Condition (i) implies that $\left\{w_{n}\right\}$ is bounded. Since $K$ is bounded and $u_{n} \in S\left(w_{n}\right) \subset$ $K\left(w_{n}\right),\left\{u_{n}\right\}$ is a bounded sequence, and hence $\left\{\left(w_{n}, u_{n}\right)\right\}$ is bounded.

By the reflexivity of $V$ and $X$, there exists a weakly convergent subsequence of $\left\{\left(w_{n}, u_{n}\right)\right\}$. Without loss of generality, we can assume that $w_{n} \rightarrow w_{0}$ in $V$ and $u_{n} \rightarrow u_{0}$ in $X$ as $n \rightarrow+\infty$.

Since $U$ is closed and convex, $U$ is a weakly closed set and so $w_{0} \in U$. By the assumptions, we know that $K\left(w_{n}\right)$ Mosco-converges to $K\left(w_{0}\right)$ and so $u_{0} \in K\left(w_{0}\right)$. According to Definition 4, there exists $u_{n}^{\prime} \in K\left(w_{n}\right)$ such that $u_{n}^{\prime} \rightarrow u_{0}$. By $u_{n} \in S\left(w_{n}\right)$, we know that $u_{n} \in K\left(w_{n}\right)$ such that

$$
\left\langle A u_{n}+B w_{n}, v-u_{n}\right\rangle_{X}+f\left(u_{n}, v\right)+J^{\circ}\left(\gamma u_{n} ; \gamma v-\gamma u_{n}\right) \geq 0, \quad \forall v \in K\left(w_{n}\right),
$$

and so

$$
\left\langle A u_{n}+B w_{n}, u_{n}^{\prime}-u_{n}\right\rangle_{X}+f\left(u_{n}, u_{n}^{\prime}\right)+J^{\circ}\left(\gamma u_{n} ; \gamma u_{n}^{\prime}-\gamma u_{n}\right) \geq 0 .
$$

Since $B$ is strongly continuous from the weak topology of $V$ to the topology of $X^{*}$, $B w_{n} \rightarrow B w_{0}$. Moreover, note that $A$ is bounded. Without loss of generality, we can 
assume that $A u_{n} \rightarrow u^{*}$. By the hypotheses, (4.1) implies that

$$
\begin{aligned}
\limsup _{n \rightarrow \infty}\left\langle A u_{n}, u_{n}-u_{0}\right\rangle_{X}= & \limsup _{n \rightarrow \infty}\left\langle A u_{n}, u_{n}-u_{0}\right\rangle_{X}+\lim _{n \rightarrow \infty}\left\langle A u_{n}, u_{0}-u_{n}^{\prime}\right\rangle_{X} \\
& +\lim _{n \rightarrow \infty}\left\langle B w_{n}, u_{n}-u_{n}^{\prime}\right\rangle_{X} \\
= & \limsup _{n \rightarrow \infty}\left\langle A u_{n}+B w_{n}, u_{n}-u_{n}^{\prime}\right\rangle_{X} \\
\leq & \limsup _{n \rightarrow \infty}\left(f\left(u_{n}, u_{n}^{\prime}\right)+J^{\circ}\left(\gamma u_{n} ; \gamma u_{n}^{\prime}-\gamma u_{n}\right)\right) \\
\leq & \lim _{n \rightarrow \infty} f\left(u_{n}, u_{n}^{\prime}\right)+\limsup _{n \rightarrow \infty} J^{\circ}\left(\gamma u_{n} ; \gamma u_{n}^{\prime}-\gamma u_{n}\right) \\
\leq &
\end{aligned}
$$

It follows from $\left(H_{A}\right)$ and Remark 1(i) that $A$ is pseudomonotone. Fixing any $v^{\prime} \in$ $K\left(w_{0}\right)$, we have

$$
\left\langle A u_{0}, u_{0}-v^{\prime}\right\rangle_{X} \leq \liminf _{n \rightarrow \infty}\left\langle A u_{n}, u_{n}-v^{\prime}\right\rangle_{X} .
$$

According to $w_{n} \rightarrow w_{0}$ and condition (ii), there exists $v_{n} \in K\left(w_{n}\right)$ such that $v_{n} \rightarrow v^{\prime}$. Since $A u_{n} \rightarrow u^{*}$, it follows that

$$
\begin{aligned}
\liminf _{n \rightarrow \infty}\left\langle A u_{n}, u_{n}-v^{\prime}\right\rangle_{X} & =\liminf _{n \rightarrow \infty}\left\langle A u_{n}, u_{n}\right\rangle_{X}+\lim _{n \rightarrow \infty}\left\langle A u_{n},-v^{\prime}\right\rangle_{X} \\
& =\liminf _{n \rightarrow \infty}\left\langle A u_{n}, u_{n}\right\rangle_{X}+\left\langle u^{*},-v^{\prime}\right\rangle_{X} \\
& =\liminf _{n \rightarrow \infty}\left\langle A u_{n}, u_{n}\right\rangle_{X}+\lim _{n \rightarrow \infty}\left\langle A u_{n},-v_{n}\right\rangle_{X} \\
& =\liminf _{n \rightarrow \infty}\left\langle A u_{n}, u_{n}-v_{n}\right\rangle_{X} .
\end{aligned}
$$

Thus,

$$
\begin{aligned}
& \left\langle A u_{0}, u_{0}-v^{\prime}\right\rangle_{X}+\left\langle B w_{0}, u_{0}-v^{\prime}\right\rangle_{X}+f\left(v^{\prime}, u_{0}\right)+J^{\circ}\left(\gamma u_{0} ; \gamma u_{0}-\gamma v^{\prime}\right) \\
\leq & \liminf _{n \rightarrow \infty}\left\langle A u_{n}, u_{n}-v^{\prime}\right\rangle_{X}+\lim _{n \rightarrow \infty}\left\langle B w_{n}, u_{n}-v_{n}\right\rangle_{X} \\
& +\liminf _{n \rightarrow \infty} f\left(v_{n}, u_{n}\right)+\lim _{n \rightarrow \infty} J^{\circ}\left(\gamma u_{n} ; \gamma u_{n}-\gamma v^{\prime}\right) \\
= & \liminf _{n \rightarrow \infty}\left\langle A u_{n}, u_{n}-v_{n}\right\rangle_{X}+\lim _{n \rightarrow \infty}\left\langle B w_{n}, u_{n}-v_{n}\right\rangle_{X} \\
& +\liminf _{n \rightarrow \infty} f\left(v_{n}, u_{n}\right)+\lim _{n \rightarrow \infty} J^{\circ}\left(\gamma u_{n} ; \gamma u_{n}-\gamma v_{n}\right) \\
\leq & \liminf _{n \rightarrow \infty}\left\langle A u_{n}+B w_{n}, u_{n}-v_{n}\right\rangle_{X}+f\left(v_{n}, u_{n}\right)+J^{\circ}\left(\gamma u_{n} ; \gamma u_{n}-\gamma v_{n}\right) .
\end{aligned}
$$

This implies that

$$
\left\langle A u_{0}+B w_{0}, v^{\prime}-u_{0}\right\rangle_{X}+f\left(u_{0}, v^{\prime}\right)+J^{\circ}\left(\gamma u_{0} ; \gamma v^{\prime}-u_{0}\right) \geq 0, \quad \forall v^{\prime} \in K\left(w_{0}\right) .
$$

Therefore, $u_{0} \in S\left(w_{0}\right)$.

Since $\mathcal{I}(w, u)$ is a weakly l.s.c. function, it follows from (2.5) that

$$
\mathcal{J}\left(w_{0}, u_{0}\right) \leq \liminf _{n \rightarrow \infty} \mathcal{I}\left(w_{n}, u_{n}\right)=\beta(0),
$$


and so

$$
\mathcal{J}\left(w_{0}, u_{0}\right)=\beta(0) .
$$

Therefore, $-\infty<\beta(0)<+\infty$. This completes the proof.

Theorem 3. Assume that all the hypotheses of Theorem 2 are satisfied. If P satisfies $\left(P_{i}\right),\left(P_{i i}\right),\left(P_{i i i}\right)$, then the zero duality gap property (2.6) holds.

Proof. From Theorem 2, $-\infty<\beta(0)<+\infty$, By Lemma 3, we only need to prove that the perturbation function $\beta$ is 1.s.c. at the origin.

On the contrary, assume that there exists an $\varepsilon_{0}>0$ such that

$$
\liminf _{y \rightarrow 0} \beta(y) \leq \beta(0)-\varepsilon_{0} .
$$

Then, there exists a sequence $\left\{y_{k}\right\} \subset \mathbb{R}, w_{k} \in U$ and $u_{k} \in K_{w_{k}}\left(y_{k}\right)$ such that

$$
\mathcal{J}\left(w_{k}, u_{k}\right) \leq \beta(0)-\frac{1}{2} \varepsilon_{0}, \quad k=1, \ldots
$$

hold.

From the proof of Theorem 2, the sequence $\left\{\left(w_{k}, u_{k}\right)\right\}$ is bounded and we can assume that $w_{k} \rightarrow w_{0} \in V$ and $u_{k} \rightarrow u_{0} \in X$ as $k \rightarrow+\infty$. Similarly, we can also get $u_{0} \in S\left(w_{0}\right)$, and so $u_{0} \in K_{w_{0}}(0)$. Since $\mathcal{I}(w, u)$ ia weakly 1.s.c. function, it follows that

$$
\beta(0) \leq \mathcal{J}\left(w_{0}, u_{0}\right) \leq \liminf _{k \rightarrow \infty} \mathcal{J}\left(w_{k}, u_{k}\right) \leq \beta(0)-\frac{1}{2} \varepsilon_{0},
$$

which is impossible. Therefore, $\beta$ is l.s.c. at the origin. The proof is complete.

\section{Corollaries}

In this section, we consider a special case in which $f(u, v)=\varphi(v)-\varphi(u)$ for all $u, v \in X$.

Find $w^{*} \in U$ and $u \in S\left(w^{*}\right)$ such that

$$
\left\{\begin{array}{l}
\min \mathcal{J}(u, w) \\
\text { subject to }(w, u) \in U \times S^{\prime}(w),
\end{array}\right.
$$

where $S^{\prime}(w)$ is the set of solutions to the following quasi-hemivariational inequality corresponding to $w$ : find $u \in K(w)$ such that

$$
\langle A u+B w, v-u\rangle_{X}+\varphi(v)-\varphi(u)+J^{\circ}(\gamma u ; \gamma v-\gamma u) \geq 0, \quad \forall v \in K(w),
$$

where $\varphi: X \rightarrow \mathbb{R} \cup\{+\infty\}$ is a convex and 1.s.c. function such that $K_{\varphi}=K(U) \cap$ $\operatorname{dom} \varphi \neq \varnothing$.

For any $w \in U$, we define

$$
\begin{gathered}
g_{w}^{\prime}(u)=\sup _{v \in K(w)}\langle A u+B w, v-u\rangle_{X}+\varphi(v)-\varphi(u)+J^{\circ}(\gamma u ; \gamma v-\gamma u), \\
K_{w}^{\prime}(y)=\left\{u \in K(w): g_{w}^{\prime}(u) \leq y\right\}
\end{gathered}
$$


Thus, $g_{w}^{\prime}(u) \geq 0$ and $K_{w}^{\prime}(0)=\left\{u \in K(w): g_{w}^{\prime}(u)=0\right\}=S^{\prime}(w)$. Define the perturbation function $\beta^{\prime}$ as

$$
\beta^{\prime}(y)=\inf _{w \in U, u \in K_{w}^{\prime}(y)} g(w, u) .
$$

It is easy to see that $\beta^{\prime}(0)$ is the optimal value of problem (5.1).

Definition 6. Let $P: \mathbb{R} \times \mathbb{R}_{+} \rightarrow \mathbb{R}$ be a function. A nonlinear Lagrangian function $L_{p}: U \times K(U) \times \mathbb{R}_{+} \rightarrow \mathbb{R}$ for problem (5.1) is defined as

$$
L_{P}^{\prime}(w, u, d)=P\left(\mathcal{J}(w, u), d g_{w}^{\prime}(u)\right) .
$$

The function

$$
F_{P}^{\prime}(d)=\inf _{w \in U, u \in K(w)} L_{P}^{\prime}(w, u, d)
$$

is called its nonlinear Lagrangian dual function. The equality

$$
\beta^{\prime}(0)=\sup _{d \in \mathbb{R}_{+}} F_{P}^{\prime}(d)
$$

is called the zero duality gap property.

Consider the following hypothesis on functional $\varphi$.

$\left(H_{\varphi}\right): \varphi: X \rightarrow \mathbb{R} \cup\{+\infty\}$ is a convex and 1.s.c. function such that $K_{\varphi} \neq \varnothing$. $\left(H_{1}\right):$

$$
\lim _{u \in X,\|u\|_{X} \rightarrow \infty} \frac{\langle A u, u\rangle_{X}+\inf _{\eta_{u} \in \partial J(\gamma u)}\left\langle\eta_{u}, \gamma u\right\rangle_{E}+\varphi(u)-\varphi(0)}{\|u\|_{X}}=+\infty .
$$

We have the following results.

Theorem 4. Assume that $\left(H_{K}\right),\left(H_{A}\right),\left(H_{J}\right),\left(H_{\gamma}\right),\left(H_{\varphi}\right),\left(H_{1}\right)$ are satisfied. Then $S^{\prime}(w) \neq \varnothing$.

Theorem 5. Assume that $\left(H_{K}\right),\left(H_{A}\right),\left(H_{J}\right),\left(H_{\gamma}\right),\left(H_{\varphi}\right)$ are satisfied. Suppose that, in addition, $\varphi$ is continuous, $K: U \rightarrow 2^{X}$ is bounded, $\mathcal{I}: U \times K(U) \rightarrow \mathbb{R}$ is weakly l.s.c. function, $B: U \rightarrow X^{*}$ is strongly continuous from the weak topology of $V$ to the topology of $X^{*}$ and the following conditions are satisfied

(i) for any $w \in U, u \in K(w), \lim _{\|w\| \rightarrow+\infty} \mathcal{I}(w, u)=+\infty$,

(ii) for all $w_{n} \in U$ with $w_{n} \rightarrow w, K\left(w_{n}\right)$ Mosco-converges to $K(w)$.

Then $-\infty<\beta^{\prime}(0)<+\infty$.

Theorem 6. Assume that all the hypotheses of Theorem 5 are satisfied. If $P$ satisfies $\left(P_{i}\right),\left(P_{i i}\right),\left(P_{i i i}\right)$, then the zero duality gap property (5.2) holds. 


\section{ACKNOWLEDGEMENT}

The work is supported by the Natural Science Foundation of Guangxi Province (No. 2019GXNSFBA185005), the Start-up Project of Scientific Research on Introducing talents at school level in Guangxi University for Nationalities (No. 2019KJQD04) and the Xiangsihu Young Scholars Innovative Research Team of Guangxi University for Nationalities (No. 2019RSCXSHQN02).

\section{REFERENCES}

[1] D. Bertsekas, Convex optimization theory. Belmont: Athena Scientific, 2009.

[2] F. Clarke, Optimization and Nonsmooth Analysis. New York: Wiley, 1983.

[3] K. Fan, "Some properties of convex sets related to fixed point theorems," Math. Ann., vol. 266, pp. 519-537, 1984.

[4] C. J. Jiang and B. Zeng, "Continuous dependence and optimal control for a class of variational-hemivariational inequalities," Appl. Math. Optim., vol. 82, pp. 637-656, 2020, doi: 10.1007/s00245-018-9543-4.

[5] D. Kinderlehrer and G. Stampacchia, An introduction to variational inequalities and their applications. New York: Wiley, 1983.

[6] Z. Liu, S. Migórski, and B. Zeng, "Existence results and optimal control for a class of quasi mixed equilibrium problems involving the $(f, g, h)$-quasimonotonicity," Appl. Math. Optim., vol. 79, pp. 257-277, 2019.

[7] Z. Liu and B. Zeng, "Optimal control of generalized quasi-variational hemivariational inequalities and its applications," Appl. Math. Optim., vol. 72, pp. 305-323, 2015.

[8] Z. Liu and B. Zeng, "Existence results for a class of hemivariational inequalities involving the stable $(g, f, \alpha)$-quasimonotonicity," Topological Methods in Nonlinear Analysis, vol. 47, pp. 195$217,2016$.

[9] S. Migórski, "Optimal control of a class of boundary hemivariational inequalities of hyperbolic type," Dynamic Continuous Discrete Impulsive Systems, Series B, vol. 2003, pp. 159-164, 2003.

[10] S. Migórski, "A note on optimal control problem for a hemivariational inequality modeling fluid flow," Discrete and Continuous Dynamical Systems, vol. 2013, pp. 533-542, 2013.

[11] S. Migórski and A. Ochal, "Optimal control of parabolic hemivariational inequalities," J. Glob. Optim., vol. 17, pp. 285-300, 2000.

[12] S. Migórski, A. Ochal, and M. Sofonea, Nonlinear Inclusions and Hemivariational Inequalities: Models and Analysis of Contact Problems. New York: Springer, 2013.

[13] S. Migórski, A. Ochal, and M. Sofonea, "A class of variational-hemivariational inequalities in reflexive Banach spaces," Journal of Elasticity, vol. 127, pp. 151-178, 2017.

[14] S. Migórski and B. Zeng, "Convergence of solutions to inverse problems for a class of variationalhemivariational inequalities," Discrete and Continuous Dynamical Systems-B, vol. 23, pp. 44774498, 2018.

[15] S. Migórski and B. Zeng, "On convergence of solutions to history-dependent variationalhemivariational inequalities," J. Math. Anal. Appl., vol. 471, pp. 496-518, 2019.

[16] U. Mosco, "Convergence of convex sets and of solutions of variational inequalities," Advances in Mathematics, vol. 3, pp. 510-585, 1969.

[17] P. Panagiotopoulos, Hemivariational inequalities, Applications in Mechanics and Engineering. Berlin: Springer, 1993.

[18] P. Panagiotopoulos, "Hemivariational inequality and Fan-variational inequality, new applications and results," Atti. Sem. Mat. Fis. Univ. Modena, vol. XLIII, pp. 159-191, 1995. 
[19] J. Park and J. Jeong, "Optimal control of hemivariational inequalities with delays," Taiwanese J. Math., vol. 15, pp. 433-447, 2011.

[20] R. Rockafellar and R. Wets, Variational Analysis. Berlin: Springer, 1998.

[21] A. Rubinov, X. Huang, and X. Yang, "The zero duality gap property and lower-semi continuity of the perturbation function," Math. Oper. Res., vol. 27, pp. 775-791, 2001.

[22] M. Sofonea, "Optimal control of a class of variational-hemivariational inequalities in reflexive banach spaces," Appl. Math. Optim., vol. 79, pp. 621-646, 2019, doi: 10.1007/s00245-017-94500 .

[23] G. Tang and N. Huang, "Existence theorems of the variational-hemivariational inequalities," $J$. Glob. Optim., vol. 56, pp. 605-622, 2013.

[24] Z. Wang, Z. Chen, Z. Chen, and S. Yao, "Lagrangian methods for optimal control problems governed by a mixed quasi-variational inequality," Optimization Letters, vol. 12, pp. 1357-1371, 2018.

[25] Z. Wang and N. Huang, "The existence results for optimal control problems governed by quasivariational inequalities in reflexive banach spaces," Taiwanese J. Math., vol. 16, pp. 1221-1243, 2012.

[26] R. Wangkeeree and P. Preechasilp, "Existence theorems of the hemivariational inequality governed by a multi-valued map perturbed with a nonlinear term in banach spaces," J. Glob. Optim., vol. 57, pp. 1447-1464, 2013.

[27] X. Yang and X. Huang, "A nonlinear lagrangian approach to constrained optimization problems," SIAM J. Control Optim., vol. 14, pp. 1119-1144, 2001.

[28] E. Zeidler, Nonlinear Functional Analysis and Its Applications, II/B, Nonlinear Monotone Operators. New York: Springer, 1990.

[29] B. Zeng and S. Migórski, "Variational-hemivariational inverse problems for unilateral frictional contact," Applicable Anal., vol. 99, pp. 293-312, 2020, doi: 10.1080/00036811.2018.1491037.

[30] Y. Zhang and Y. He, "On stably quasimonotone hemivariational inequalities," Nonlinear Anal., vol. 74, pp. 3324-3332, 2011.

[31] Y. Zhou, X. Yang, and K. Teo, "The existence results for optimal control problems governed by a variational inequality,” J. Math. Anal. Appl., vol. 321, pp. 595-608, 2006.

[32] Y. Zhou, X. Yang, and K. Teo, "Optimal control problems governed by a variational inequality via nonlinear lagrangian methods," Optimization, vol. 55, pp. 187-203, 2006.

Authors' addresses

Fengzhen Long

Nanjing University of Sciences and Technology, School of Science, Nanjing 210094, P.R. China

Biao Zeng

(corresponding author) Guangxi University for Nationalities, Faculty of Mathematics and Physics, Nanning 530006, P.R. China

E-mail address: biao_zeng@163.com 